\title{
Depletion of RUVBL2 in Human Cells Confers Moderate Sensitivity to Anticancer Agents
}

\author{
Mayumi Miyamoto-Matsubara', Yi Han', Koji Ono ${ }^{1}$, Ming-Zhang Xie ${ }^{1}$, Amir MH Salem ${ }^{1,2}$, Mahmoud I Shoulkamy ${ }^{1,3}$, Toshiaki Nakano ${ }^{1}$ and \\ Hiroshi Ide ${ }^{1 *}$
}

${ }^{1}$ Department of Mathematical and Life Sciences, Graduate School of Science, Hiroshima University, Higashi-Hiroshima 739-8526, Japan

${ }^{2}$ Department of Pathology, Medical Research Division, National Research Centre, El-Bohouth St., Dokki, Giza 12311, Egypt

${ }^{3}$ Department of Zoology, Biological Science Building, Faculty of Science, Minia University, Minia 61519, Egypt

\begin{abstract}
Background: Many anticancer agents kill cancer cells by inducing lethal damage in DNA, but the capacity of DNA repair of cells reduce the therapeutic efficacy of anticancer agents. RuvB-like (RUVBL) 2 is part of large protein complexes such as TIP60 and INO80 that are involved in chromatin remodeling and DNA damage responses and repair. Relatively few studies have investigated the role of RUVBL2 in the survival of cells after exposure to anticancer agents.
\end{abstract}

Methods: We depleted RUVBL2 in human MRC5-SV cells by small interfering (si) RNA and assessed the sensitivity of the cells to chemotherapeutic anticancer agents including cisplatin (cisPt), 2'-deoxy-5-azacytidine (azadC), and mitomycin C (MMC), and to physical DNA-damaging agents including X-rays.

Results: The knockdown efficiency with $10 \mathrm{nM}$ siRUVBL2 was $80 \%$ on day 3 post-transfection, and knockdown $(>65 \%)$ persisted on day 6 . The cell growth slowed significantly due to depletion of RUVBL2 when compared to mock- and control siRNA-treated cells, indicating that RUVBL2 is essential for the proliferation of cells. The RUVBL2-depleted cells were moderately sensitive to cisPt, azadC, and X-rays. The increase in the sensitivity to MMC was marginal.

Conclusion: Depletion of RUVBL2 in cells confers moderate sensitivity to anticancer agents and X-rays, presumably through partial impairment of the homologous recombination repair of DNA double-strand break intermediates formed directly or indirectly by anticancer agents or X-rays. Further studies are necessary to clarify the exact role of RUVBL2 in this process.

Keywords: DNA repair; Chromatin remodeling; Cell survival; Anticancer drugs; X-rays

\section{Introduction}

RuvB-like 1 (RUVBL1, also known as RVB1, TIP49A and Pontin) and RuvB-like 2 (RUVBL2, also known as RVB2, TIP49B and Reptin) belong to the family of ATPases associated with various cell activities (AAA+ ATPase), and are part of large protein complexes that are involved in chromatin remodeling, transcription regulation, DNA damage responses and repair, and the biogenesis of small nucleolar ribonucleoproteins [1,2]. RUVBL1 and RUVBL2 (RUVBLs) share about $45 \%$ identity and $60 \%$ similarity in the amino acid sequence and are homologous to the prokaryotic RuvB helicase [3,4]. Remarkably, RUVBL1 depletion leads to down regulation of RUVBL2, and vice versa [5]. RUVBLs also interact with oncogenes such as c-Myc and $\beta$-catenin and are implicated in cancer $[4,6]$. Since RUVBLs participate in many aspects of cellular functions, links between these functions are currently being studied extensively.

In human cells, RUVBLs serve as the essential components of the TIP60 histone acetyltransferase complex. TIP60 is recruited to DNA damage sites $[7,8]$ and plays multiple roles in DNA damage responses [9]. After treatment with various DNA-damaging agents, RUVBL1 is required for the dephosphorylation of phosphorylated histone $\mathrm{H} 2 \mathrm{AX}$, a marker of DNA double-strand breaks (DSBs), through the histone acetyltransferase activity of TIP60 [8]. Likewise, depletion of RUVBL2 increases the persistence of phosphorylated histone H2AX upon treatment with X-rays [10]. Apart from the pathway involving the TIP60 complex, RUVBLs regulate the abundance and activity of phosphatidylinositol 3-kinases such as ataxia telangiectasia mutated
(ATM), ATM- and Rad3-related (ATR) kinase, and DNA-dependent protein kinase (DNA-PK) that sense and activate the DNA damage signal [11]. RUVBLs are also components of the INO80 chromatin remodeling complex that is recruited to the DSB site. A recent study has shown that the mammalian INO80 complex mediates DSB repair through its role in DNA end resection [12]. The ATP-dependent helicase activity of RUVBLs demonstrated in vitro may be involved in the processing of resected DNA ends $[13,14]$. Depletion of RUVBLs in human cells impairs the recruitment of the RAD51 recombinase to the DSB site following DNA end resection [15]. Consistent with the finding, it has been shown that the functional inactivation or depletion of TIP60 that contains RUVBLs in mouse and human cells impairs the recruitment of RAD51 to the DSB site [16].

The aforementioned studies have afforded mechanistic insights into the function of human RUVBLs in DNA damage responses and repair. However, few studies have investigated the role of RUVBLs in

*Corresponding author: Hiroshi Ide, Department of Mathematical and Life Sciences, Graduate School of Science, Hiroshima University, HigashiHiroshima 739-8526, Japan, Tel: +81-82-424-7457; Fax: +81-82-424-7457; E-mail: ideh@hiroshima-u.ac.jp

Received July 29, 2014; Accepted October 16, 2014; Published October 20 2014

Citation: Matsubara MM, Han Y, Ono K, Xie MZ, Salem AMH, et al. (2014) Depletion of RUVBL2 in Human Cells Confers Moderate Sensitivity to Anticancer Agents. J Cancer Sci Ther 6: 440-445. doi:10.4172/1948-5956.1000306

Copyright: $\odot 2014$ Matsubara MM, et al. This is an open-access article distributed under the terms of the Creative Commons Attribution License, which permits unrestricted use, distribution, and reproduction in any medium, provided the original author and source are credited. 
the survival of human cells after exposure to DNA-damaging agents, although decreased cell survival through impaired DNA damage responses and repair is among the major end points of DNA damage. It is also noteworthy that many anticancer agents kill cancer cells by inducing lethal damage in DNA, but the capacity of DNA repair of cells reduce the therapeutic efficacy of anticancer agents $[17,18]$. To the best of our knowledge, enhancement of sensitivity to mitomycin $\mathrm{C}$ (MMC) by the depletion of RUVBL1 [15] and that to ultraviolet light (UV) by the depletion of RUVBL2 $[19,20]$ have only been reported for human cells. We therefore thought it would be useful to analyze the effect of down-regulation of RUVBLs on cell survival upon treatment with chemical DNA-damaging agents such as anticancer drugs and physical DNA-damaging agents such as X-rays. In the present study, we depleted RUVBL2 in human MRC5-SV cells by small interfering (si) RNA and assessed the sensitivity of the cells to anticancer drugs including cisplatin (cisPt), 2'-deoxy-5-azacytidine (azadC), MMC, and to X-rays.

\section{Materials and Methods}

\section{Cell culture and overexpression of RUVBL2}

DNA repair-proficient MRC5-SV cells, a human fetal lung fibroblast cell line transfected with SV40, were used for depletion of RUVBL2 [21,22], since mutations in DNA repair genes that are frequently found in cancer cells [23] may obscure the direct effect of RUVBL2 depletion. HEK293T cells were used for overexpression of RUVBL2. MRC5-SV and HEK293T cells were cultured in Dulbecco's modified Eagle's medium (Nissui) supplemented with 10\% inactivated fetal bovine serum (Biological Industries), L-glutamine $(584 \mu \mathrm{g} / \mathrm{ml})$ and kanamycin $(20 \mu \mathrm{g} / \mathrm{ml})$ in a humidified incubator at $37^{\circ} \mathrm{C}$ with a $5 \%$ $\mathrm{CO}_{2}$ atmosphere. For the overexpression of RUVBL2, HEK293T cells were transfected with pCMV6-XL5-RUVBL2 or PCMV6-AC-RUVBL2 (both from Origene) using Lipofectamine LTX and Plus reagent (Invitrogen) according to the method provided by the manufacturer. After transfection, cells were cultured for $72 \mathrm{~h}$, recovered and assayed for protein expression. Chinese hamster ovary $(\mathrm{CHO})$ cells proficient (AA8) and deficient (51D1) in homologous recombination (HR) were cultured in $\alpha$-minimum essential medium (Wako) supplemented with $10 \%$ inactivated fetal bovine serum [24].

\section{RNA interference}

MRC5-SV cells at ca. 50\% confluence in a $10 \mathrm{~cm}$ dish were transfected with RUVBL2 siRNA (2-30 nM) or control siRNA (Invitrogen) using Lipofectamine 2000 (Invitrogen) according to the method provided by the manufacturer. One day after transfection, the cells were divided into four plates, incubated for up to 5 days and assayed for the depletion of RUVBL2. The target sequences of RUVBL2 siRNA were 5'-gctccacgcagtacatgaaggagta-3' (siRUVBL2-1), 5'-gagatccagattgatcgaccagcaa-3' (siRUVBL2-2) and 5'-ccagcttggtgtgccggaaacgcaa-3' (siRUVBL2-3). Two negative-control siRNAs with high (siCONT-H) and medium (siCONT-M) GC contents were also used.

\section{Western blot analysis}

MRC5-SV cells were transfected with siRNA as described above, and cells were recovered from the plates after 3 and 6 days. Cells were resuspended in cold RIPA buffer ( $25 \mathrm{mM}$ Tris- $\mathrm{HCl}$ (pH 7.6), $150 \mathrm{mM}$ $\mathrm{NaCl}, 1 \% \mathrm{NP}-40,1 \%$ sodium deoxycholate, and $0.1 \% \mathrm{SDS}$ ) supplemented with $1 \mathrm{mM}$ PMSF for $10 \mathrm{~min}$, sonicated twice for $2 \mathrm{~s}$ each and then sedimented at $22,000 \times g$ for $20 \mathrm{~min}$ at $4^{\circ} \mathrm{C}$. The recovered supernatant was used as whole cell extracts (WCEs). The protein concentration was measured with the BCA protein assay kit (Pierce). WCEs were separated by electrophoresis on a $7.5 \%$ SDS-polyacrylamide gel, and proteins were transferred to a PDVF membrane on a Trans-Blot Turbo transfer system (BIO-RAD). The membrane was washed with TBST (20 $\mathrm{mM}$ Tris- $\mathrm{HCl}$ ( $\mathrm{pH} 7.6$ ), $150 \mathrm{mM} \mathrm{NaCl}$, and $0.1 \%$ Tween 20), blocked with $1 \%$ BSA in TBST and then washed again with TBST. RUVBL2 was proved with anti-RUVBL2 antibodies (Abcam EPR4146, 1:5000 dilution). Primary antibodies were detected with HRP-conjugated goat anti-rabbit IgG antibodies (KPL 474-1516, 1:5000 dilution). $\beta$-Tubulin was used as a loading control and was detected with anti- $\beta$-tubulin antibodies (Sigma T8328, 1:5000 dilution) and HRP-conjugated antimouse IgG antibodies (Santa Cruz Biotechnology sc-2005, 1:2000 dilution). Finally, the membrane was incubated with the ECL Western Blotting Substrate (Promega), and chemiluminescence was detected on a ChemiDoc XRS+ system (BIO-RAD). The sizes of bands were estimated relative to the Precision Plus Protein WesternC Standard (BIO-RAD) run in parallel.

\section{Cell proliferation and survival}

MRC5-SV cells were transfected with siRNA as described above. One day after transfection, the cells were divided into four plates and incubated for up to 5 days. The cells were stained with trypan blue and the numbers of viable and dead cells were counted. Survival assays with MRC5-SV cells were performed as follows: One day after transfection with siRNA, cells were recovered, seeded in a dish and incubated for 24 h. Cells were treated with cisPt, azadC, or MMC (all from Wako) for 3 $\mathrm{h}$ (cisPt and MMC) or $24 \mathrm{~h}$ (azadC). Alternatively, cells were irradiated with X-rays generated by an OM-303M X-ray generator $(\mathrm{OHMiC})$ at a dose rate of $1.14 \mathrm{~Gy} / \mathrm{min}$. The treated cells were further incubated in fresh medium for 7-8 days, and colonies with more than 50 cells were scored. The surviving fraction was calculated as $\mathrm{N} / \mathrm{N}_{0}$, where $\mathrm{N}$ and $\mathrm{N}_{0}$ are the numbers of colonies for DNA damaging agent-treated and untreated cells, respectively. Survival assays with $\mathrm{CHO}$ cells were performed as described for MRC5-SV cells except that siRNA treatment was omitted.

\section{Results}

\section{Detection of RUVBL2 by western blot analysis}

We optimized the conditions for immunodetection of endogenous RUVBL2 prior to its depletion by siRNA. In the Western blot analysis of RUVBL2, the WCEs from MRC5-SV cells gave rise to two bands at around $50 \mathrm{kDa}$ (expected size $=51.2 \mathrm{kDa}$, Figure 1A). Comparison with the mobility of the RUVBL2 protein overexpressed from pCMV6-XL5RUVBL2 or pCMV6-AC-RUVBL2 revealed that the upper band was attributable to endogenous RUVBL2. The cross-reacting protein in the lower band remains to be identified. The band intensity of endogenous RUVBL2 was proportional to the amount of WCEs up to $15 \mu \mathrm{g}$ (Figure $1 B)$. From the result, we used $10 \mu \mathrm{g}$ of WCEs for the analysis of the down-regulation of RUVBL2.

\section{Depletion of RUVBL2 by siRNA}

Three different siRNAs (siRUVBL2-1, -2 and -3) were designed to target the coding sequence of the RUVBL2 mRNA. The siRNAs were transfected into MRC5-SV cells, and the depletion of RUVBL2 was analysed by Western blotting at 3 and 6 days after transfection. The knockdown efficiency of RUVBL2 increased with increasing the concentrations of siRNA (2-30 nM, data not shown), and nearmaximal silencing was achieved with $10 \mathrm{nM}$ siRUVBL2-1, -2 and -3 . The knockdown efficiency with $10 \mathrm{nM}$ siRUVBL2s was $80 \%$ on day 3 
Citation: Matsubara MM, Han Y, Ono K, Xie MZ, Salem AMH, et al. (2014) Depletion of RUVBL2 in Human Cells Confers Moderate Sensitivity to Anticancer Agents. J Cancer Sci Ther 6: 440-445. doi:10.4172/1948-5956.1000306

A

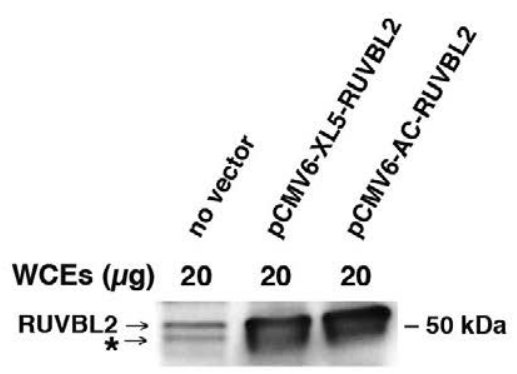

B
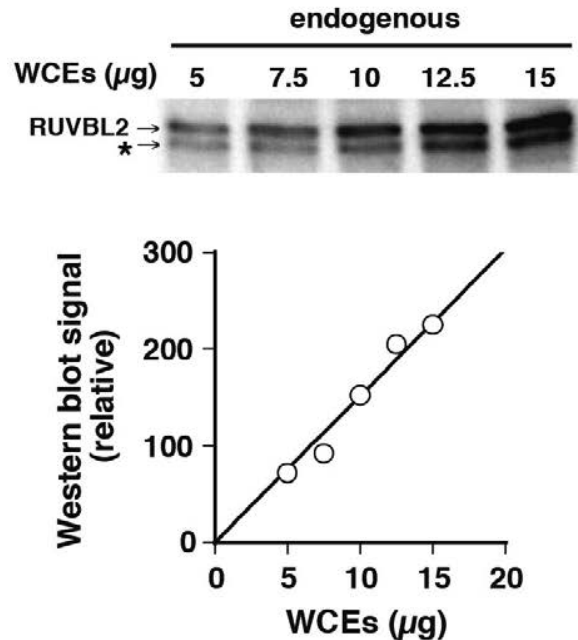

Figure 1: Detection of endogenous RUVBL2. (A) Detection of RUVBL2 by Western blot analysis. WCEs of MRC5-SV cells without transfection and HEK293T cells transfected with a RUVBL2 expression vector (pCMV6-XL5RUVBL2 or pCMV6-AC-RUVBL2) were separated by SDS-polyacrylamide gel electrophoresis. RUVBL2 protein on a blotted membrane was detected by anti-RUVBL2 antibodies. (B) Correlation between the amount of WCEs of MRC5-SV cells and the Western blot signal of endogenous RUVBL2. Upper panel: Western blot; lower panel: correlation between the amount of WCEs and the Western blot signal (average from two experiments). A cross-reacting protein in the lower band, which remains to be identified, is indicated by the asterisks in $(A)$ and $(B)$.

post-transfection, and knockdown $(>65 \%)$ persisted on day 6 (Figure 2).

\section{Inhibition of cell proliferation by the depletion of RUVBL2}

RUVBLs are essential for the cell proliferation of yeast $[3,25]$, C. elegans [26], drosophila [27] and xenopus [28]. We asked whether this is also true for MRC5-SV cells. Figure 3 shows the growth curves of MRC5-SV cells transfected with RUVBL2 siRNAs (10 nM). The cell growth slowed significantly due to depletion of RUVBL2 when compared to mock- and control siRNA-treated cells, indicating that RUVBL2 is essential for the proliferation of MRC5-SV cells. The result is consistent with the previous observation that silencing of RUVBL2 (and RUVBL1) impedes the proliferation of HeLa cells [29]. More recently it was shown that silencing of RUVBL2 blocks the progression of human hepatocellular carcinoma in xenografts without inducing significant apoptosis [30]. We also compared the fractions of viable and dead cells by using trypan blue dye exclusion at 3 or 4 days after transfection. The fraction of viable cells was $80-90 \%$ for mock-, siCONT- and siRNA-treated cells, showing no systematic differences between the treatments. Thus, the slow proliferation of the siRNA- treated cells was due to growth retardation per se and not the increase in the fraction of dead cells.

\section{Sensitivity of RUVBL2-depleted cells to anticancer agents and $\mathrm{X}$-rays}

In order to obtain insight into the role of human RUVBL2 in protecting the genome, we analyzed the survival of RUVBL2-depleted MRC5-SV cells using colony formation assays after treatment with cisPt, azadC, MMC, and X-rays. Figure 4 shows the typical survival curves of knockdown cells when RUVBL2 was depleted by siRUVBL2-2. The
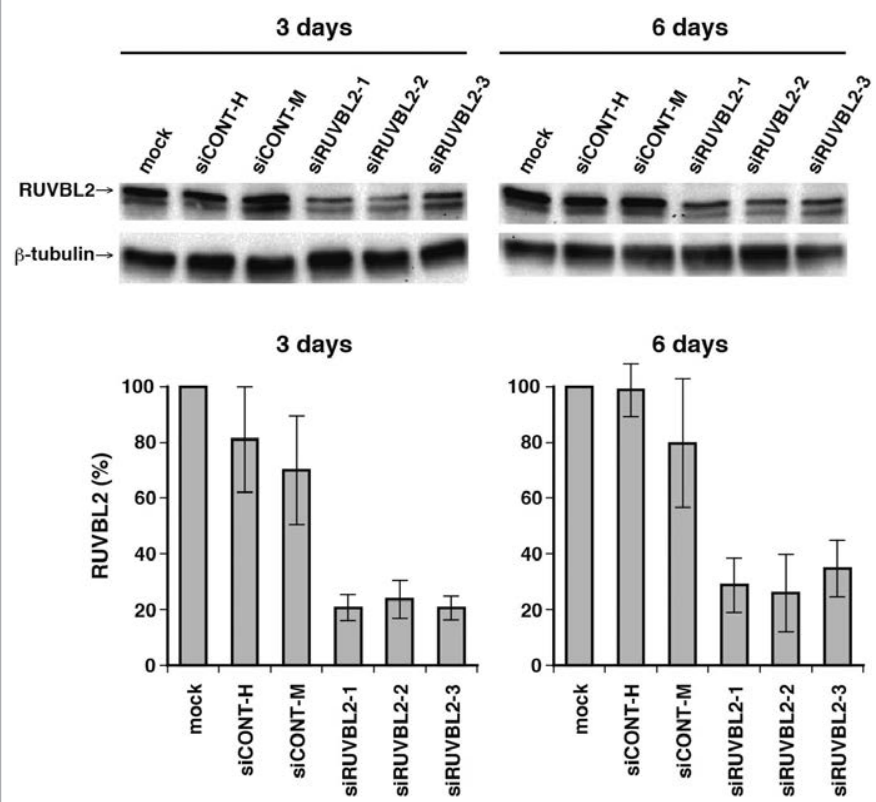

Figure 2: Depletion of RUVBL2 by siRNA. siRNAs for RUVBL2 (10 nM siRUVBL2-1, -2 and -3 ) or control siRNAs (10 nM siCONT-H and -M) were transfected into MRC5-SV cells, and the depletion of RUVBL2 was analysed by Western blotting at 3 and 6 days after transfection. Upper panels: typical Western blots for RUVBL2 and $\beta$-tubulin (loading control); Lower panels: depletion of RUVBL2 in siRNA-treated cells relative to mock-treated cells. Data points are means of 3 independent experiments with standard deviation.

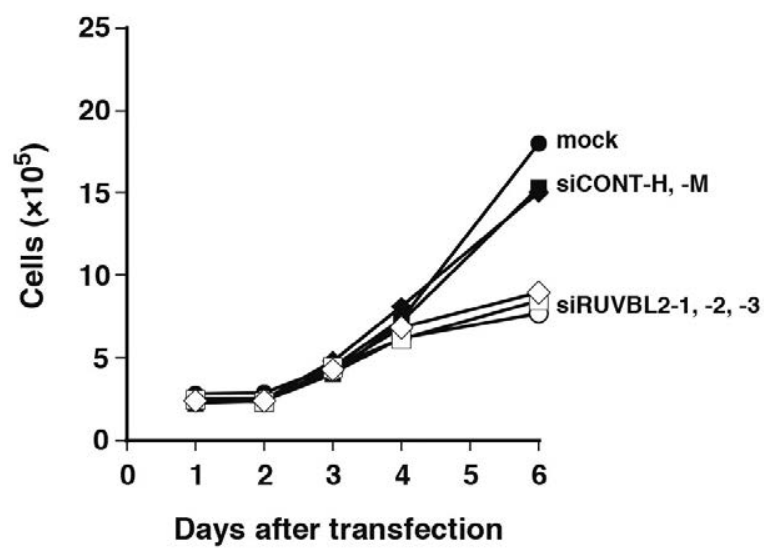

Figure 3: Inhibition of cell proliferation by the depletion of RUVBL2. siRNAs for RUVBL2 (10 nM siRUVBL2-1, -2 and -3, open symbols) or control siRNAs (10 nM siCONT-H and -M, closed square and diamond) were transfected into MRC5-SV cells and the number of cells was counted on the indicated days. The data points are the average from two experiments. 

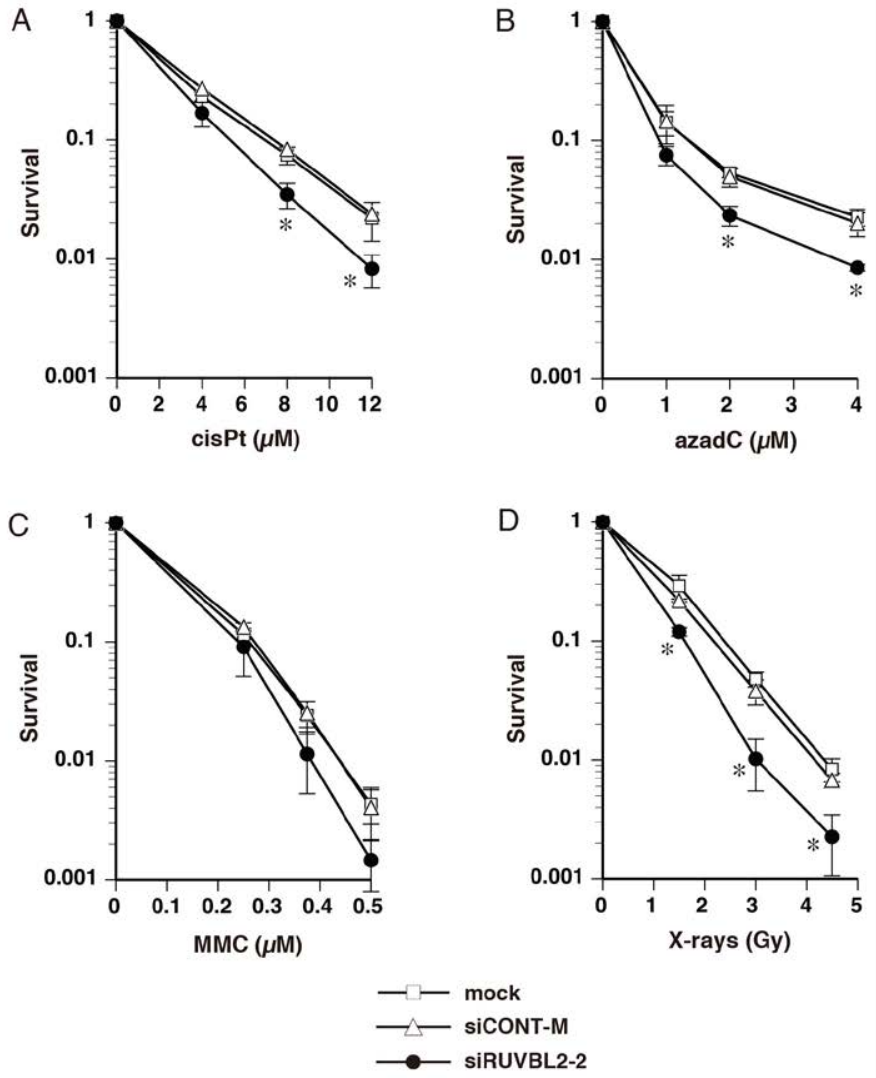

Figure 4: Sensitivity of RUVBL2-depleted cells to anticancer agents and X-rays. siRNA for RUVBL2 (10 nM siRUVBL2-2, closed circle) or control siRNA (10 nM siCONT-M, open triangle) was transfected into MRC5-SV cells and incubated as described in Materials and Methods. Two days after transfection, the cells were treated with cisPt $(\mathrm{A})$, azadC $(\mathrm{B}), \mathrm{MMC}(\mathrm{C})$, or $X$-rays (D). The treated cells were further incubated in fresh medium for 7-8 days, and colonies were scored to calculate the surviving fraction. Data points are means of 3 independent experiments with standard deviation. Statistically significant differences in survival ( $t$-test, $p<0.05$ ) between siRUVBL2-2-treated and siCONT-M-treated cells are indicated by asterisks.

RUVBL2-depleted cells were moderately sensitive to cisPt, azadC, and X-rays (Figure 4A, B, and C). Conversely, the RUVBL2-depleted cells were not sensitive to MMC: the difference in the sensitivity between RUVBL2-depleted and control siRNA (siCONT-M)-treated cells was not statistically significant (Figure 4C). The survival of cells treated with other siRNAs (siRUVBL2-1 and siRUVBL2-3) was also analyzed using the same anticancer agents and X-rays: the results were similar to those obtained with siRUVBL2-2 (data not shown). It should be noted that we scored the colonies with more than 50 cells in the cell survival assays (Figure 4). In a parallel experiment, we examined the colonies with more than 20 cells and found that the cell survival was essentially the same as that obtained with colonies with more than 50 cells, indicating that the cell survival data were not affected by growth retardation by the depletion of RUVBL2.

\section{Sensitivity of HR-deficient cells to anticancer agents and X-rays}

RUVBLs play a role in recombination repair of DNA damage as described in introduction. To compare the impacts of depletion of RUVBL2 and HR on the sensitivity to anticancer agents and X-rays, we measured the survival of HR (RAD51D)-deficient CHO cells to cisPt,
azadC, MMC, and X-rays. RAD51D-deficient cells exhibited more pronounced sensitivity to the anticancer agents and X-rays than those deficient in RUVBL2 (Figure 5). In contrast to the RUVBL2-depleted cells (Figure 3), the HR-deficient CHO cells showed only mild growth retardation as compared to wild type AA8 cells, which is consistent with the previous observation [24].

\section{Discussion}

In the present study we have shown that depletion of RUVBL2 renders human MRC5-SV cells moderately sensitive to chemotherapeutic anticancer agents including cisPt and azadC, and to X-rays (Figure 4). The present data thus expand our knowledge regarding the role of RUVBLs in cell survival after exposure to anticancer agents and X-rays. It has been demonstrated previously that depletion of RUVBL1 and RUVBL2 from human cells confers sensitivity to MMC [15] and UV [19,20], respectively. However, our data showed that depletion of RUVBL2 from MRC5-SV cells conferred no statistically significant sensitivity to MMC (Figure 4C).

It is well established that X-rays induce DSBs as the major lethal lesions. azadC is incorporated into DNA and covalently traps the reaction intermediate of DNA cytosine methyltransferase, giving rise to DNA-protein cross-links (DPCs) [31]. DPCs block DNA replication and transcription [32,33] and hence constitute the major lethal lesions upon treatment with azadC. cisPt is a bifunctional DNA-damaging
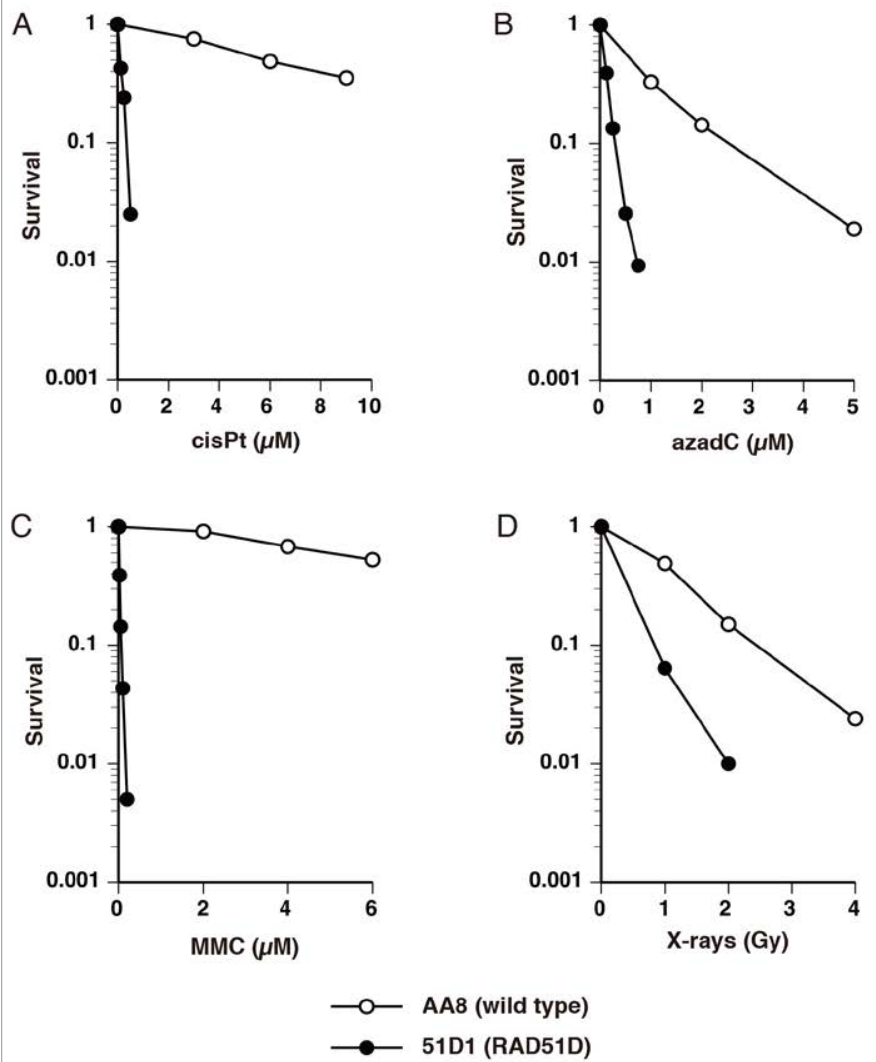

Figure 5: Sensitivity of $\mathrm{HR}$-deficient $\mathrm{CHO}$ cells to anticancer agents and X-rays. AA8 (wild type, open circle) and 51D1 (deficient in RAD51D, closed circle) cells were treated with cisPt (A), azadC (B), MMC (C), or X-rays (D). The treated cells were further incubated in fresh medium for 6 days, and colonies were scored to calculate the surviving fraction. The data points are the average from two experiments. 
Citation: Matsubara MM, Han Y, Ono K, Xie MZ, Salem AMH, et al. (2014) Depletion of RUVBL2 in Human Cells Confers Moderate Sensitivity to Anticancer Agents. J Cancer Sci Ther 6: 440-445. doi:10.4172/1948-5956.1000306

agent and reacts with two guanine residues in the same DNA strand or two opposing DNA strands, yielding intrastrand cross-links and interstrand cross-links (ICLs) [34]. DPCs can also form when cisPt reacts with guanine and a proximal protein. ICLs inhibit the unwinding of duplex DNA upon DNA replication and hence can constitute the lethal lesions upon treatment with cisPt. Although the spectra of lethal lesions associated with anticancer agents used in this study differ considerably, DSBs are commonly involved as intermediates both in the reactivation of the replication fork stalled by DPCs [35] and the repair of ICLs [36], where DSB intermediates resulting from DPCs and ICLs are repaired by HR [35-37]. Thus, the sensitivity to cisPt, azadC, and $\mathrm{X}$-rays could be increased if the depletion of RUVBL2 compromises the repair of DSB intermediates via HR. This mechanism is consistent with the observations made in mammalian cells that depletion of RUVBLs or inactivation of the TIP60 histone acetyltransferase containing RUVBLs impairs the recruitment of RAD51, the key recombinase in HR, to DSBs $[15,16]$. In addition, depletion of RUVBL2 may compromise the activity of the INO80 chromatin remodeling complex that is involved in the DNA end resection of DSBs [12]. In yeast, INO80 and SWR1 chromatin remodeling complexes contain the orthologs of RUVBLs and are suggested in chromatin remodeling in the vicinity of DSBs [3840]. Functional inactivation of either yeast INO80 or SWR1 complex renders cells sensitive to DNA-damaging agents including methyl methanesulfonate, $\mathrm{UV}$, ionizing radiation and hydroxyurea to varying extents [41-43]. It is therefore possible that the function of RUVBLs in the survival of cells exposed to DNA-damaging agents is at least partially conserved from yeast to human.

We noticed that RUVBL2-depleted MRC5-SV cells were less sensitive to anticancer agents and $\mathrm{X}$-rays than were $\mathrm{CHO}$ cells deficient in HR (Figure 5). The residual RUVBL2 in the knockdown cells (Figure 2) may be sufficient to partially support the repair of DSB intermediates by HR. This could also be true for cell proliferation (Figure 3 ). Alternatively, RUVBL2 or complexes containing RUVBL2 such as TIP60 and INO80 may act as a promoting but not essential factor in repair. In addition, it has been reported that the human INO80 complex containing RUVBLs positively regulates the expression of RAD54B and XRCC3, which are involved in HR, and may contribute indirectly to DSB repair [44].

In summary, RUVBL2 is involved in the survival of human cells after exposure to a wide range of anticancer agents and X-rays, but further studies are necessary to clarify the exact role of RUVBLs in this process.

\section{Acknowledgements}

We thank Larry $\mathrm{H}$. Thompson for the generous gift of AA8 and 51D1 cells. This work was supported in part by a Grant-in-Aid for Scientific Research on Innovative Areas from the Ministry of Education, Culture, Sports, Science and Technology of Japan (H.I.) and a Grant-in-Aid for Young Scientists (B) from the Japan Society for the Promotion of Science (M.M.).

The authors declare that there are no conflicts of interest.

\section{References}

1. Jha S, Dutta A (2009) RVB1/RVB2: running rings around molecular biology Mol Cell 34: 521-533.

2. Grigoletto A, Lestienne P, Rosenbaum J (2011) The multifaceted proteins Reptin and Pontin as major players in cancer. Biochim Biophys Acta 1815: 147-157.

3. Kanemaki M, Kurokawa Y, Matsu-ura T, Makino Y, Masani A, et al. (1999) TIP49b, a new RuvB-like DNA helicase, is included in a complex together with another RuvB-like DNA helicase, TIP49a. J Biol Chem 274: 22437-22444.
4. Wood MA, McMahon SB, Cole MD (2000) An ATPase/helicase complex is an essential cofactor for oncogenic transformation by c-Myc. Mol Cell 5: 321-330.

5. Haurie V, Menard L, Nicou A, Touriol C, Metzler P, et al. (2009) Adenosine triphosphatase pontin is overexpressed in hepatocellular carcinoma and coregulated with reptin through a new posttranslational mechanism. Hepatology 50: 1871-1883

6. Feng Y, Lee N, Fearon ER (2003) TIP49 regulates beta-catenin-mediated neoplastic transformation and T-cell factor target gene induction via effects on chromatin remodeling. Cancer Res 63: 8726-8734.

7. Ikura T, Tashiro S, Kakino A, Shima H, Jacob N, et al. (2007) DNA damagedependent acetylation and ubiquitination of $\mathrm{H} 2 \mathrm{AX}$ enhances chromatin dynamics. Mol Cell Biol 27: 7028-7040.

8. Jha S, Shibata E, Dutta A (2008) Human Rvb1/Tip49 is required for the histone acetyltransferase activity of Tip60/NuA4 and for the downregulation of phosphorylation on H2AX after DNA damage. Mol Cell Biol 28: 2690-2700.

9. Sun Y, Jiang X, Price BD (2010) Tip60: connecting chromatin to DNA damage signaling. Cell Cycle 9: 930-936.

10. Higgins GS, Prevo R, Lee YF, Helleday T, Muschel RJ, et al. (2010) A small interfering RNA screen of genes involved in DNA repair identifies tumor-specific radiosensitization by POLQ knockdown. Cancer Res 70: 2984-2993.

11. Izumi N, Yamashita A, Iwamatsu A, Kurata R, Nakamura H, et al. (2010) AAA+ proteins RUVBL1 and RUVBL2 coordinate PIKK activity and function in nonsense-mediated mRNA decay. Sci Signal 3: ra27.

12. Gospodinov A, Vaissiere T, Krastev DB, Legube G, Anachkova B, et al. (2011) Mammalian Ino80 mediates double-strand break repair through its role in DNA end strand resection. Mol Cell Biol 31: 4735-4745

13. Papin C, Humbert O, Kalashnikova A, Eckert K, Morera S, et al. (2010) 3'- to 5 DNA unwinding by TIP49b proteins. FEBS J 277: 2705-2714.

14. Seeber A, Hauer M, Gasser SM (2013) Nucleosome remodelers in doublestrand break repair. Curr Opin Genet Dev 23: 174-184.

15. Gospodinov A, Tsaneva I, Anachkova B (2009) RAD51 foci formation in response to DNA damage is modulated by TIP49. Int J Biochem Cell Biol 41: 925-933.

16. Murr R, Loizou JI, Yang YG, Cuenin C, Li H, et al. (2006) Histone acetylation by Trrap-Tip60 modulates loading of repair proteins and repair of DNA doublestrand breaks. Nat Cell Biol 8: 91-99.

17. Madhusudan S, Middleton MR (2005) The emerging role of DNA repair proteins as predictive, prognostic and therapeutic targets in cancer. Cancer Treat Rev 31: 603-617.

18. Madhusudan S, Hickson ID (2005) DNA repair inhibition: a selective tumour targeting strategy. Trends Mol Med 11: 503-511.

19. Ni L, Saeki M, Xu L, Nakahara H, Saijo M, et al. (2009) RPAP3 interacts with Reptin to regulate UV-induced phosphorylation of $\mathrm{H} 2 \mathrm{AX}$ and DNA damage. J Cell Biochem 106: 920-928.

20. Wu S, Shi Y, Mulligan P, Gay F, Landry J, et al. (2007) A YY1-INO80 complex regulates genomic stability through homologous recombination-based repair. Nat Struct Mol Biol 14: 1165-1172.

21. Huschtscha LI, Holliday R (1983) Limited and unlimited growth of SV40 transformed cells from human diploid MRC-5 fibroblasts. J Cell Sci 63: 77-99.

22. Kannouche $\mathrm{P}$, Broughton BC, Volker M, Hanaoka F, Mullenders LH, et al (2001) Domain structure, localization, and function of DNA polymerase eta defective in xeroderma pigmentosum variant cells. Genes Dev 15: 158-172.

23. Negrini S, Gorgoulis VG, Halazonetis TD (2010) Genomic instability--an evolving hallmark of cancer. Nat Rev Mol Cell Biol 11: 220-228.

24. Hinz JM, Tebbs RS, Wilson PF, Nham PB, Salazar EP, et al. (2006) Repression of mutagenesis by Rad51D-mediated homologous recombination. Nucleic Acids Res 34: 1358-1368.

25. Qiu XB, Lin YL, Thome KC, Pian P, Schlegel BP, et al. (1998) An eukaryotic RuvB-like protein (RUVBL1) essential for growth. J Biol Chem 273: 2778627793.

26. Sheaffer KL, Updike DL, Mango SE (2008) The Target of Rapamycin pathway antagonizes pha-4/FoxA to control development and aging. Curr Biol 18: 1355 1364. 
Citation: Matsubara MM, Han Y, Ono K, Xie MZ, Salem AMH, et al. (2014) Depletion of RUVBL2 in Human Cells Confers Moderate Sensitivity to Anticancer Agents. J Cancer Sci Ther 6: 440-445. doi:10.4172/1948-5956.1000306

27. Bauer A, Chauvet S, Huber O, Usseglio F, Rothbächer U, et al. (2000) Pontin52 and reptin52 function as antagonistic regulators of beta-catenin signalling activity. EMBO J 19: 6121-6130.

28. Etard C, Gradl D, Kunz M, Eilers M, Wedlich D (2005) Pontin and Reptin regulate cell proliferation in early Xenopus embryos in collaboration with c-Myc and Miz-1. Mech Dev 122: 545-556.

29. Watkins NJ, Lemm I, Ingelfinger D, Schneider C, Hossbach M, et al. (2004) Assembly and maturation of the U3 snoRNP in the nucleoplasm in a large dynamic multiprotein complex. Mol Cell 16: 789-798.

30. Ménard L, Taras D, Grigoletto A, Haurie V, Nicou A, et al. (2010) In vivo silencing of Reptin blocks the progression of human hepatocellular carcinoma in xenografts and is associated with replicative senescence. J Hepatol 52: 681 689.

31. Santi DV, Norment A, Garrett CE (1984) Covalent bond formation between a DNA-cytosine methyltransferase and DNA containing 5-azacytosine. Proc Natl Acad Sci U S A 81: 6993-6997.

32. Nakano T, Miyamoto-Matsubara M, Shoulkamy MI, Salem AM, Pack SP, et al. (2013) Translocation and stability of replicative DNA helicases upon encountering DNA-protein cross-links. J Biol Chem 288: 4649-4658.

33. Nakano T, Ouchi R, Kawazoe J, Pack SP, Makino K, et al. (2012) T7 RNA polymerases backed up by covalently trapped proteins catalyze highly error prone transcription. J Biol Chem 287: 6562-6572.

34. Kartalou M, Essigmann JM (2001) Recognition of cisplatin adducts by cellular proteins. Mutat Res 478: 1-21.

35. Nakano T, Katafuchi A, Matsubara M, Terato H, Tsuboi T, et al. (2009) Homologous recombination but not nucleotide excision repair plays a pivotal role in tolerance of DNA-protein cross-links in mammalian cells. J Biol Chem 284: $27065-27076$
36. Kim H, D'Andrea AD (2012) Regulation of DNA cross-link repair by the Fancon anemia/BRCA pathway. Genes Dev 26: 1393-1408.

37. Ide H, Shoulkamy MI, Nakano T, Miyamoto-Matsubara M, Salem AM (2011) Repair and biochemical effects of DNA-protein crosslinks. Mutat Res 711: 113122.

38. Morrison AJ, Highland J, Krogan NJ, Arbel-Eden A, Greenblatt JF, et al (2004) INO80 and gamma-H2AX interaction links ATP-dependent chromatin remodeling to DNA damage repair. Cell 119: 767-775.

39. Downs JA, Allard S, Jobin-Robitaille O, Javaheri A, Auger A, et al. (2004) Binding of chromatin-modifying activities to phosphorylated histone $\mathrm{H} 2 \mathrm{~A}$ at DNA damage sites. Mol Cell 16: 979-990.

40. van Attikum H, Fritsch O, Gasser SM (2007) Distinct roles for SWR1 and INO80 chromatin remodeling complexes at chromosomal double-strand breaks. EMBO J 26: 4113-4125.

41. Shen X, Mizuguchi G, Hamiche A, Wu C (2000) A chromatin remodelling complex involved in transcription and DNA processing. Nature 406: 541-544.

42. van Attikum H, Fritsch O, Hohn B, Gasser SM (2004) Recruitment of the INO80 complex by $\mathrm{H} 2 \mathrm{~A}$ phosphorylation links ATP-dependent chromatin remodeling with DNA double-strand break repair. Cell 119: 777-788.

43. Mizuguchi G, Shen X, Landry J, Wu WH, Sen S, et al. (2004) ATP-driven exchange of histone $\mathrm{H} 2 \mathrm{AZ}$ variant catalyzed by SWR1 chromatin remodeling complex. Science 303: 343-348.

44. Park EJ, Hur SK, Kwon J (2010) Human INO80 chromatin-remodelling complex contributes to DNA double-strand break repair via the expression of Rad54B and XRCC3 genes. Biochem J 431: 179-187. 